\title{
Electrode-Level State Estimation in Lithium-ion Batteries via Kalman Decomposition
}

\author{
Dong Zhang, Member, IEEE, Luis D. Couto, Member, IEEE, and Scott J. Moura, Member, IEEE
}

\begin{abstract}
Lithium-ion battery electrode-level online state estimation using high-fidelity nonlinear electrochemical models remains a key challenge. This is particularly due to weak observability inherited from the complex model structure, even for reduced-order electrochemical models. This manuscript presents a systematic and rigorous strategy to analyze the local observability of a single particle model (SPM) with both electrodes, which is commonly known to be locally unobservable from current-voltage measurements. Estimating the essential states, e.g. state of charge (SOC) and solid-phase lithium surface concentration, is crucial for battery charge and health monitoring since different degradation mechanisms affect each electrode individually. In this manuscript, the proposed observability analysis approach based on the Kalman decomposition enables provably convergent estimates. Ultimately, using the observability analysis, we propose a state estimator based on the nonlinear SPM dynamics and prove estimation error system stability. The observability analysis and state estimation scheme exploits the conservation of lithium property. Simulations demonstrate the effectiveness of the electrode-level state estimator as opposed to the cell-level estimator.
\end{abstract}

Index Terms-Lithium-ion Batteries, State Estimation, Observability, Kalman Decomposition.

\section{INTRODUCTION}

$\mathbf{E}$ NERGY storage is a critical enabling technology for electrified transportation and clean variable renewables integration. In this context, lithium-ion (Li-ion) batteries are the technology of choice, given their high efficiency and energy/power density [1]. The internal electrochemical states of Li-ion batteries should be carefully monitored in order to prevent over-charge and over-discharge. Most internal states of the battery electrochemical models cannot be directly measured, and therefore algorithms should be implemented for estimation. This task is difficult because the states are typically weakly observable and have nonlinear relationships with the output measurements. A battery management system (BMS) that estimates the state of different battery components would be a leap forward for the advanced control of battery systems.

Model-based estimation of Li-ion batteries have been widely considered in the literature [2]. The estimation methods can

Luis D. Couto would like to thank the Wiener-Anspach Foundation for its financial support.

Dong Zhang and Scott J. Moura are with Department of Civil and Environmental Engineering, University of California, Berkeley, CA 94720 USA (e-mail: dongzhr@berkeley.edu, smoura@berkeley.edu).

Luis D. Couto is with Department of Control Engineering and System Analysis, Université Libre de Bruxelles, B-1050 Brussels, Belgium (email: Icoutome@ulb.ac.be). be classified according to the mathematical models at their core. The first models - data-driven models - do not have any physical interpretation and require a large amount of training data [3], [4]. Meanwhile, equivalent circuit models rely on an electric circuit analogy for batteries, and thus do not explicitly represent relevant electrochemical processes [5], [6]. Electrochemical models are the most complex, and they directly characterize the electrochemical behavior of Li-ion batteries [7], [8]. Even if high-order electrochemical models serve as a benchmark (e.g., the Doyle-Fuller-Newman model), reduced-order models are needed for real-time implementations in estimation and control. The most popular reduced-order electrochemical model is the single particle model (SPM) [9], due to its intriguing balance between model accuracy and simplicity.

A common model reduction for electrochemical modelbased observer designs is to consider only one electrode. The reason behind the single-electrode approximation is the weak observability when estimating the lithium concentration in both electrodes, from the cell's output voltage [10]. The one-electrode simplification can be achieved through two approaches: (i) assume an open-loop model for one electrode; or (ii) assume the moles of lithium are conserved. In the first approach, one electrode provides a pseudo voltage measurement and the other electrode state is estimated [9], [11], [12]. This method requires a proper initialization of the electrode in open-loop in order to ensure that the state estimates of the electrode in closed-loop converge to the true values. In the second approach, lithium balance adds one algebraic constraint that can be exploited for observer design. This is, by far, the most prolific research direction, giving rise to Kalman filters [10], sliding mode observers [13], robust observers [14], and partial differential equation (PDE) backstepping observers [15], where the state of one electrode depends linearly on the other. In contrast to the first approach, the second one trades off proper initialization of the second electrode with correct knowledge of the lithium balance. Note that all the aforementioned estimators effectively estimate the state of only one of the electrodes.

Recently, estimating states in both electrodes has been gaining attentions. This task is rewarding in real-time monitor of operational conditions in both electrodes, but extremely challenging due to weak observability. Recent works have been attempting to tackle this issue via three different paths. The first one is to use extra measurements such as temperature sensors [16] or expansion sensors [17], besides current and voltage, to improve observability. The additional instrumen- 
tation, however, might be inconvenient in practice since it increases the cost and complexity of the sensing network. The second option is to use interconnected state observers that deal with the estimation of each electrode separately but exchange information to guarantee closed-loop convergence [18], [19]. Although these observers provide convergent estimates, they do so at the expense of complex interconnected observer structures. Yet a third way relies on lithium material balance in the electrode phase to design either PDE backstepping observers [9], [20] or Luenberger observers [21]. Ultimately, although relevant and illuminating, the first two approaches do so at the cost of extra hardware sensors and added mathematical design complexity. The last one builds on battery physical intuition and explores the conservation of lithium in the electrodes, but does not provide any analysis and guarantee on observability with both electrodes. In this manuscript, we specifically address the research gaps in the third approach by proposing an elegant and mathematically rigorous framework to tackle the lack of observability issue when simultaneously estimating states in both electrodes in SPM. This task is achieved by developing a comprehensive and rigorous scheme utilizing the concept of Kalman decomposition, which attempts to transform a system into a structure in which the new system is decomposed into observable and un-observable sub-systems. The transformed system enables a systematic way to analyze the local observability, and ultimately a nonlinear state estimator with asymptotic convergence is designed in the transformed domain to reconstruct the essential states in both electrodes. Note that even though the proposed analysis similarly requires knowledge of the moles of lithium, it performs observability analysis which facilitates the electrode-level state observer designs in an elegant and rigorous manner based on Kalman decomposition.

Notation. Throughout the manuscript, $\mathbb{R}^{n}$ denotes a coordinate space over the real numbers with dimension $n . \mathbb{N}^{+}$is the space containing all strictly positive integers. For a matrix $A, A_{i, j}$ represents the element located at the $i$-th row and $j$ th column. For a vector $x, x_{k}$ indicates the $k$-th entry. $\|\cdot\|$ indicates the $L_{2}$ Euclidean norm. $\mathbb{I}$ is an identity matrix.

\section{MOdEL DEscription}

In the full-order electrochemical model [7], Li-ion transports in the solid and electrolyte phases. The key idea of the SPM is that the solid phase of each electrode can be modeled as a single spherical particle, and Li-ion concentration in electrolyte phase is assumed to be constant. The intercalation process of SPM is modeled through a linear diffusion PDE over a spherical domain, which describes the lithium concentration in an electrode active material particle,

$$
\frac{\partial c_{s}^{ \pm}}{\partial t}(r, t)=D_{s}^{ \pm}\left[\frac{\partial^{2} c_{s}^{ \pm}}{\partial r^{2}}(r, t)+\frac{2}{r} \frac{\partial c_{s}^{ \pm}}{\partial r}(r, t)\right],
$$

with Neumann boundary conditions

$$
\frac{\partial c_{s}^{ \pm}}{\partial r}(0, t)=0, \quad \frac{\partial c_{s}^{ \pm}}{\partial r}\left(R_{s}^{ \pm}, t\right)=-m^{ \pm} u(t),
$$

where $c_{s}^{+}(r, t):\left[0, R_{s}^{+}\right] \times[0, \infty) \rightarrow \mathbb{R}$ and $c_{s}^{-}(r, t):$ $\left[0, R_{s}^{-}\right] \times[0, \infty) \rightarrow \mathbb{R}$ map the radial position and time to
TABLE I

Single Particle Model Symbol Description

\begin{tabular}{ccc}
\hline \hline Symbols & Description & Units \\
\hline$a^{ \pm}$ & Specific interfacial surface area & {$\left[\mathrm{m}^{2} / \mathrm{m}^{3}\right]$} \\
$A$ & Cell cross sectional area & {$\left[\mathrm{m}^{2}\right]$} \\
$c_{e}^{0}$ & Li-ion conc. in electrolyte & {$\left[\mathrm{mol} / \mathrm{m}^{3}\right]$} \\
$c_{s}^{ \pm}$ & Solid phase Li-ion concentration & {$\left[\mathrm{mol} / \mathrm{m}^{3}\right]$} \\
$c_{s s}^{ \pm}$ & Li-ion conc. at particle surface & {$\left[\mathrm{mol} / \mathrm{m}^{3}\right]$} \\
$\bar{c}_{s}^{ \pm}$ & Volume-averaged Li-ion conc. & {$\left[\mathrm{mol} / \mathrm{m}^{3}\right]$} \\
$c_{s, \max }^{ \pm}$ & Max Li-ion conc. in solid phase & {$\left[\mathrm{mol} / \mathrm{m}^{3}\right]$} \\
$\theta_{s s}^{ \pm}$ & Normalized Li-ion surface conc. & {$\left[\mathrm{mol} / \mathrm{m}^{3}\right]$} \\
$\bar{\theta}_{s}^{ \pm}$ & Normalized volume-averaged conc. & {$\left[\mathrm{mol} / \mathrm{m}^{3}\right]$} \\
$D_{s}^{ \pm}$ & Solid phase diffusion coefficient & {$\left[\mathrm{m}^{2} / \mathrm{sec}\right]$} \\
$F$ & Faraday's constant & {$[\mathrm{C} / \mathrm{mol}]$} \\
$I$ & Applied current & {$[\mathrm{A}]$} \\
$k^{ \pm}$ & Charge transfer reaction rate & {$\left[\mathrm{A} \cdot \mathrm{m}^{2.5} / \mathrm{mol}{ }^{1.5}\right]$} \\
$L^{ \pm}$ & Electrode thickness & {$[\mathrm{m}]$} \\
$n_{\mathrm{Li}, \mathrm{s}}$ & Total Li in the solid phase & {$[\mathrm{mol}]$} \\
$r$ & Radial coordinate & {$[\mathrm{m}]$} \\
$R$ & Universal gas constant & {$[\mathrm{J} / \mathrm{mol}-\mathrm{K}]$} \\
$R_{f}$ & Contact film resistance & {$[\mathrm{Ohm}]$} \\
$R_{s}^{ \pm}$ & Particle radius & {$[\mathrm{m}]$} \\
$t$ & Time & {$[\mathrm{Second}]$} \\
$U^{ \pm}$ & Open circuit potential & {$[\mathrm{V}]$} \\
$\alpha^{ \pm}$ & Charge transfer coefficient & {$[-]$} \\
$\varepsilon_{s}^{ \pm}$ & Volume fraction of solid phase & {$[-]$} \\
\hline \hline
\end{tabular}

solid phase lithium concentration in the positive electrode and negative electrode, respectively. Symbol $u(t)$ is the input, and it represents either an intercalation current $I(t)$ or an intercalation current density $J^{ \pm}(t)$, and coefficients $m^{+}$and $m^{-}$are factors determined by the choice of $u(t)$ [22]:

$$
J^{+}(t)=-\frac{1}{F a^{+} L^{+}} I(t), \quad J^{-}(t)=\frac{1}{F a^{-} L^{-}} I(t) .
$$

The output terminal voltage $V(t)$ is a function of solid phase surface concentrations, open circuit potentials, electric overpotentials, and the Butler-Volmer kinetics:

$$
\begin{aligned}
V(t)= & \delta^{+} \sinh ^{-1}\left[\frac{-\beta^{+} I(t)}{i_{0}^{+}\left(c_{s s}^{+}(t)\right)}\right]-\delta^{-} \sinh ^{-1}\left[\frac{\beta^{-} I(t)}{i_{0}^{-}\left(c_{s s}^{-}(t)\right)}\right] \\
& +U^{+}\left(c_{s s}^{+}(t)\right)-U^{-}\left(c_{s s}^{-}(t)\right)-R_{f} I(t),
\end{aligned}
$$

where $\delta^{ \pm}=R T /\left(\alpha^{ \pm} F\right), \beta^{ \pm}=1 /\left(2 a^{ \pm} A L^{ \pm}\right)$, and the exchange current density $i_{0}^{ \pm}(\cdot)$ is given by

$$
\begin{aligned}
i_{0}^{ \pm}\left(c_{s s}^{ \pm}\right) & =k^{ \pm} \sqrt{c_{e}^{0} c_{s s}^{ \pm}(t)\left(c_{s, \max }^{ \pm}-c_{s s}^{ \pm}(t)\right)}, \\
c_{s s}^{ \pm}(t) & =c_{s}^{ \pm}\left(R_{s}^{ \pm}, t\right) .
\end{aligned}
$$

$U^{+}(\cdot)$ and $U^{-}(\cdot)$ in (4) are the equilibrium potentials of the positive and negative electrode material as functions of solid phase surface concentrations. A complete notation description for the SPM can be found in Table I.

\section{OBSERVABILITY ANALYSIS}

This section provides insights on PDE model reduction in the frequency domain, and model decomposition oriented towards observability analysis in the linear sense. 


\section{A. Model Reduction}

According to existing works in [15], [23], the PDE model (1)-(2) for each electrode can be described in the frequency domain as a transcendental transfer function between the surface concentration and input,

$$
\frac{c_{s}^{ \pm}(s, r)}{U(s)}=\frac{e^{\kappa(s)\left(R_{s}^{ \pm}-r\right)}\left(e^{2 \kappa(s) r}-1\right) m^{ \pm}\left(R_{s}^{ \pm}\right)^{2}}{\left(1+R_{s}^{ \pm} \kappa(s)+e^{2 R_{s}^{ \pm} \kappa(s)}\left(R_{s}^{ \pm} \kappa(s)-1\right)\right) r},
$$

where $\kappa(s)=\sqrt{s / D_{s}^{ \pm}}$. Two physically important quantities of the SPM are surface concentration $c_{s s}^{ \pm}$and volume-averaged concentration $\bar{c}_{s}^{ \pm}$. The surface concentration plays a role in the electrochemical reaction and the volume-averaged concentration is associated with SOC of each electrode. Evaluating (7) at $r=R_{s}^{ \pm}$yields the transfer function for surface concentration,

$$
\frac{c_{s s}^{ \pm}(s)}{U(s)}=\frac{\sinh \left(\kappa(s) R_{s}^{ \pm}\right) m^{ \pm} R_{s}^{ \pm}}{R_{s}^{ \pm} \kappa(s) \cosh \left(\kappa(s) R_{s}^{ \pm}\right)-\sinh \left(\kappa(s) R_{s}^{ \pm}\right)} .
$$

One can also conveniently obtain the volume-averaged concentration by evaluating

$$
\frac{\bar{c}_{s}^{ \pm}(s)}{U(s)}=\frac{1}{4 / 3 \pi\left(R_{s}^{ \pm}\right)^{3}} \int_{0}^{R_{s}^{ \pm}} \frac{c_{s}^{ \pm}(r, s)}{U(s)}\left(4 \pi r^{2}\right) \mathrm{d} r=\frac{q^{ \pm}}{s},
$$

where $q^{ \pm}=3 D_{s}^{ \pm} m^{ \pm} / R_{s}^{ \pm}$. Next, Padé approximation is applied to the transcendental transfer function (8) and (9). The Padé approximation approximates the transcendental transfer functions as a ratio of two power series that naturally contain poles and zeros:

$$
G(s)=\frac{b_{0} s^{n}+b_{1} s^{n-1}+\cdots+b_{n-1} s+b_{n}}{s^{n}+a_{1} s^{n-1}+\cdots+a_{n-1} s+a_{n}} .
$$

The coefficients of the numerator and denominator are computed via moment matching [22], [23]. The order of the approximation is determined based on the trade-off between the number of states and desired model accuracy required to predict the frequency response of the original diffusion PDE model. The authors of [23] note that a third order truncation is often sufficient for capturing the solid-phase diffusion dynamics, particularly in electric vehicle applications.

There are essentially an infinite number of state-space realizations for (10). We choose to adopt the controllable canonical form in this study. Note the proposed approach can be universally adapted to other realizations. The controllable canonical form for a 3rd-order Padé approximation is defined in terms of the coefficients of (10) as follows,

$$
\begin{aligned}
& \dot{x}^{ \pm}=A^{ \pm} x^{ \pm}+B^{ \pm} u, \\
& c_{s s}^{ \pm}=H_{s s}^{ \pm} x^{ \pm}, \quad \bar{c}_{s}^{ \pm}=\bar{H}_{s}^{ \pm} x^{ \pm},
\end{aligned}
$$

where $x^{+}, x^{-} \in \mathbb{R}^{3}$,

$$
\begin{aligned}
x^{ \pm} & =\left[\begin{array}{l}
x_{1}^{ \pm} \\
x_{2}^{ \pm} \\
x_{3}^{ \pm}
\end{array}\right], \quad A^{ \pm}=\left[\begin{array}{ccc}
0 & 1 & 0 \\
0 & 0 & 1 \\
-a_{3}^{ \pm} & -a_{2}^{ \pm} & -a_{1}^{ \pm}
\end{array}\right], \quad B^{ \pm}=\left[\begin{array}{l}
0 \\
0 \\
1
\end{array}\right], \\
H_{s s}^{ \pm} & =\left[\begin{array}{lll}
b_{3}^{ \pm} & b_{2}^{ \pm} & b_{1}^{ \pm}
\end{array}\right], \bar{H}_{s}^{ \pm}=\left[\begin{array}{lll}
q^{ \pm} a_{2}^{ \pm} & q^{ \pm} a_{1}^{ \pm} & q^{ \pm}
\end{array}\right] .
\end{aligned}
$$

The fact that $a_{3}^{+}=a_{3}^{-}=0$ indicates that there is one pole at zero in each electrode. Thus, an open-loop observer will not guarantee asymptotic convergence. The system that concatenates dynamics of both electrodes and the voltage measurement is written as

$$
\dot{x}=A x+B u, \quad y=h(x, u),
$$

where function $h(x, u)$ is given by (4) and $x=\left[\begin{array}{ll}x^{+} & x^{-}\end{array}\right]^{\top}$, $A=\operatorname{diag}\left(A^{+}, A^{-}\right), B=\left[\begin{array}{ll}B^{+} & B^{-}\end{array}\right]^{\top}$.

It is previously noted in the literature, e.g., [10], [15], that the single particle model with both electrode dynamics is not locally observable from the cell's output voltage measurement. This conclusion can be physically interpreted from the output voltage expression (4). Intuitively, the cell voltage (4) is dependent on voltage difference from two electrodes, which guarantees the observability of the difference of the electrode voltage but does not make each electrode voltage observable. Additionally, the Jacobian of the cell voltage with respect to surface concentrations of cathode and anode are low for certain regions within the battery operational window. This low Jacobian also degenerates system observability. Furthermore, this phenomenon can be also demonstrated mathematically. If we linearize the nonlinear output equation $h(x, u)$ about the states around an equilibrium point to produce a matrix $C_{p}$, the rank of the observability matrix for the pair $\left(A, C_{p}\right)$ is less than the order of system (14), which will be further detailed in Section III-C. Hence the lithium concentration from both electrodes are not locally observable from the cell voltage output in the linear sense.

\section{B. Model Decomposition}

A Kalman decomposition transforms a linear time invariant (LTI) system into a form in which the system can be decomposed into (non)controllable and (non)observable components [24], [25]. It essentially combines the controllability canonical decomposition, where the state space is separated into the controllable subspace and its complement, and observability canonical decomposition, in which the state space is decomposed into observable and unobservable subspace. The standard Kalman decomposition makes clear the controllable and observable components of a system.

The objective of this work is to examine the observability of the SPM Padé approximation with both electrodes in a mathematically rigorous way using Kalman decomposition. Although the system (14) is nonlinear, we study the observability in the linear sense by linearizing the output function $h(x, u)$ around an equilibrium point. The next theorem sets the foundation for the observability decomposition.

Theorem 1 (Observability Decomposition [26]): Let $x \in$ $\mathbb{R}^{n}, \dot{x}=A x+B u, y=C x+D u$ be unobservable with rank of the observability matrix, $\operatorname{rank}(\mathcal{O})=d<n$. Let $P=\left[\begin{array}{ll}P_{o} & P_{u o}\end{array}\right]^{\top}$, where $P_{o}$ consists of $d$ linearly independent rows of $\mathcal{O}$, and $P_{u o}$ are added rows to complete the basis and yield a nonsingular $P$. Then $\bar{x}=P x$ transforms the system , to

$$
\begin{aligned}
{\left[\begin{array}{c}
\dot{\bar{x}}_{o} \\
\dot{\bar{x}}_{u o}
\end{array}\right] } & =\left[\begin{array}{cc}
\bar{A}_{o} & 0 \\
\bar{A}_{21} & \bar{A}_{u o}
\end{array}\right]\left[\begin{array}{c}
\bar{x}_{o} \\
\bar{x}_{u o}
\end{array}\right]+\left[\begin{array}{c}
\bar{B}_{o} \\
\bar{B}_{u o}
\end{array}\right] u, \quad \bar{x}_{o} \in \mathbb{R}^{d}, \\
y & =\left[\begin{array}{ll}
\bar{C}_{o} & 0
\end{array}\right]\left[\begin{array}{c}
\bar{x}_{o} \\
\bar{x}_{u o}
\end{array}\right]+D u .
\end{aligned}
$$


Furthermore, the pair $\left(\bar{A}_{o}, \bar{C}_{o}\right)$ is observable.

The similarity transformation $P$ in Theorem 1 preserves the stability properties of the system prior to the transformation, i.e., the system (15) also has two poles at the origin. The transformation $P$ in Theorem 1 clearly turns any LTI system into a structure in which observable and unobservable components are decoupled. Specifically, it is guaranteed that $\bar{x}_{o}$ is observable from the system input-output data, so that we are able to construct a closed-loop observer to estimate the unknown $\bar{x}_{o}$. Additionally, if the matrix $\bar{A}_{u o}$ for the unobservable part of the system is Hurwitz, i.e., all eigenvalues of $\bar{A}_{u o}$ have strict negative real part, then we could design an openloop observer for the unobservable component. Under this scenario, the system (15)-(16) is detectable. These conditions are sufficient for the reconstruction of the entire state space in $\bar{x}$-domain, which can be subsequently used to establish the estimates of $x$ by performing the inverse transformation $x=P^{-1} \bar{x}$.

\section{Observability Analysis via Kalman Decomposition}

Under equilibrium conditions with zero current, the output expression simplifies to

$$
V(t)=h(x)=U^{+}\left(c_{s s}^{+}(t)\right)-U^{-}\left(c_{s s}^{-}(t)\right) .
$$

We first analyze the observability of the nonlinear system (14) with (17) by linearizing function $h(x)$ and checking the rank of the linear observability matrix. Let the partial derivatives of $h(x)$ be $C_{p}=\mathrm{d} h / \mathrm{d} x$. Namely

$$
C_{p}=\left[\begin{array}{llllll}
\frac{\partial h}{\partial x_{1}^{+}} & \frac{\partial h}{\partial x_{2}^{+}} & \frac{\partial h}{\partial x_{3}^{+}} & \frac{\partial h}{\partial x_{1}^{-}} & \frac{\partial h}{\partial x_{2}^{-}} & \frac{\partial h}{\partial x_{3}^{-}}
\end{array}\right],
$$

where

$$
\frac{\partial h}{\partial x_{j}^{+}}=\frac{\partial U^{+}}{\partial c_{s s}^{+}} \frac{\partial c_{s s}^{+}}{\partial x_{j}^{+}}, \quad \text { and } \quad \frac{\partial h}{\partial x_{j}^{-}}=-\frac{\partial U^{-}}{\partial c_{s s}^{-}} \frac{\partial c_{s s}^{-}}{\partial x_{j}^{-}},
$$

with $j=\{1,2,3\}$. The terms $\partial U^{+} / \partial c_{s s}^{+}$and $\partial U^{-} / \partial c_{s s}^{-}$are gradients (Jacobian) of open circuit potential (OCP) function for cathode and anode, respectively. It is well known that the OCP function for anode (typically graphite) is flat, especially within 10\%-90\% anode SOC range (see, for instance, Fig. 6 in [17]). This flatness renders small numeric values for the gradient $\partial U^{-} / \partial c_{s s}^{-}$, weakening the local observability by potentially lowering the rank of the linear observability matrix. Similar observation can be made from the cathode OCP function. However, even with high OCP gradients in some regions, system (14) is still not locally observable in the linear sense because the rank of the observability matrix constructed by the pair $\left(A, C_{p}\right)$, denoted by $\mathcal{O}_{p}$, is $(n-1)$ around any equilibrium points (rank deficient). This because of the zero pole at each electrode, which causes the first and the fourth columns of matrix $A$ to be all-zero columns. Thus, the first and the fourth columns of matrix $A^{i}$, for all $i \in \mathbb{N}^{+}$, are all-zero columns. This leads to the fact that the first and the fourth elements of $C \cdot A^{i}$, for all $i \in \mathbb{N}^{+}$, are zeros. Hence, these two columns in $\mathcal{O}_{p}$ are linearly dependent.

Applying Theorem 1 to decouple system (14) for observability analysis in the linear sense would require $d=5$ linearly independent rows from the matrix $\mathcal{O}_{p}$. The special structure of $\mathcal{O}_{p}$, in which the only non-zero elements in the first and the fourth columns are offered by the first row $\left(C_{p}\right)$, requires that the transformation matrix $P$ must include the first row of $\mathcal{O}_{p}$, otherwise $P$ will not be invertible however we construct $P_{u o}$. Another intriguing point to highlight is that the element $\bar{A}_{u o} \equiv 0$ regardless of the design of $P_{u o}$. That is, the eigenvalue of $\bar{A}_{u o}$ is zero. This can be verified by declaring $P_{u o}$ as a symbolic variable in any numerical computation tool and checking the general expression of $\bar{A}=P A P^{-1}$. Hence, for the battery system (14), the transformed system (15)-(16) is not detectable, prohibiting the design of an open-loop observer for $\bar{x}_{u o}$.

Proposition 1 (Conservation of solid-phase lithium): The total moles of lithium in the solid phase is conserved [9], [21]. Mathematically,

$$
\begin{aligned}
n_{\mathrm{Li}, \mathrm{s}}=M x & =\sum_{j=\{+,-\}} \frac{\epsilon_{s}^{j} L^{j} A}{4 / 3 \pi\left(R_{s}^{j}\right)^{3}} \int_{0}^{R_{s}^{j}} c_{s}^{j}(r, t)\left(4 \pi r^{2}\right) \mathrm{d} r \\
& =\epsilon_{s}^{+} L^{+} A \bar{c}_{s}^{+}+\epsilon_{s}^{-} L^{-} A \bar{c}_{s}^{-},
\end{aligned}
$$

where $M=\left[\begin{array}{ll}M^{+} & M^{-}\end{array}\right]$with

$$
M^{ \pm}=\left[\varepsilon_{s}^{ \pm} L^{ \pm} A q^{ \pm} a_{2}^{ \pm} \quad \varepsilon_{s}^{ \pm} L^{ \pm} A q^{ \pm} a_{1}^{ \pm} \quad \varepsilon_{s}^{ \pm} L^{ \pm} A q^{ \pm}\right] .
$$

Our ultimate goal is to estimate all states in $\bar{x}$-domain, and in turn, the surface concentrations $c_{s s}^{ \pm}$and volume-averaged concentrations $\bar{c}_{s}^{ \pm}$. This cannot be accomplished if $\bar{x}_{u o}$ is not estimated or known a priori. Hence, we utilize the conservation of solid-phase lithium property to construct $P_{u o}$ by making $P_{u o}=M$. The result of such a construction ensures

$$
\bar{x}_{u o}=P_{u o} x=M x=n_{\mathrm{Li}, \mathrm{s}}
$$

is a known constant. Therefore, although $\bar{x}_{u o}$ is not observable, its temporal values are pre-determined and uniformly equal to the total moles of lithium of solid phase over time.

The analysis in this section is based on the linearized system and the observability conclusions are local. In the next section, we design an observer to estimate $\bar{x}_{o}$ using the nonlinear voltage function.

\section{Observer Design}

The dynamics for the observable component in (15) after the observability decomposition are given by

$$
\dot{\bar{x}}_{o}=\bar{A}_{o} \bar{x}_{o}+\bar{B}_{o} u, \quad y=h\left(\bar{x}_{o}, \bar{x}_{u o}, u\right),
$$

where $\bar{x}_{u o}=n_{\mathrm{Li}, \mathrm{s}}$ is a constant by construction, and the nonlinear function $h$ is the output voltage represented by the states in the transformed coordinate,

$$
\begin{aligned}
h= & \delta^{+} \sinh ^{-1}\left[\frac{-\beta^{+} I(t)}{i_{0}^{+}\left(\bar{x}_{o}, \bar{x}_{u o}\right)}\right]-\delta^{-} \sinh ^{-1}\left[\frac{\beta^{-} I(t)}{i_{0}^{-}\left(\bar{x}_{o}, \bar{x}_{u o}\right)}\right] \\
& +U^{+}\left(\bar{x}_{o}, \bar{x}_{u o}\right)-U^{-}\left(\bar{x}_{o}, \bar{x}_{u o}\right)-R_{f} I(t),
\end{aligned}
$$

where we have replaced $c_{s s}^{+}$and $c_{s s}^{-}$in (4) with

$$
\begin{aligned}
& c_{s s}^{+}=\sum_{k=1}^{6}\left(b_{3}^{+} N_{1, k}+b_{2}^{+} N_{2, k}+b_{1}^{+} N_{3, k}\right) \bar{x}_{k}, \\
& c_{s s}^{-}=\sum_{k=1}^{6}\left(b_{3}^{-} N_{4, k}+b_{2}^{-} N_{5, k}+b_{1}^{-} N_{6, k}\right) \bar{x}_{k},
\end{aligned}
$$


in which $N$ is the inverse transformation $N=P^{-1}$.

Remark 1: It is evident from (5) and (24) that function $h$ is continuously differentiable with respect to the state $\bar{x}$ within the normal battery operational region, which is a sufficient condition for Lipschitz continuity [27]. Mathematically, a Lipschitz constant with respect to the state $\bar{x}$ can be obtained by computing the infinity norm of $\partial h / \partial \bar{x}_{o}$, i.e., $\gamma=\left\|\partial h / \partial \bar{x}_{o}\right\|_{\infty}$, such that

$$
\left\|h\left(\bar{x}_{o}^{1}, \bar{x}_{u o}, u\right)-h\left(\bar{x}_{o}^{2}, \bar{x}_{u o}, u\right)\right\| \leq \gamma\left\|\bar{x}_{o}^{1}-\bar{x}_{o}^{2}\right\| .
$$

We are now positioned to propose the following observer with linear output error injection for system (23),

$$
\dot{\bar{x}}_{o}=\bar{A}_{o} \hat{\bar{x}}_{o}+\bar{B}_{o} u+L(y-\hat{y}), \quad \hat{y}=h\left(\hat{\bar{x}}_{o}, \bar{x}_{u o}, u\right),
$$

where $\hat{\bar{x}}_{o}$ is the estimate of $\bar{x}_{o}$ and gain $L$ is to be designed to ensure observer convergence. Similar design procedures for nonlinear Lipschitz system can be found in [14], [28]. Theorem 2 provides the convergence results of the observer.

Theorem 2: Consider the plant model dynamics (23) and the corresponding state observer (28). Let the error between the actual and the estimated states be $\tilde{\bar{x}}_{o}=\bar{x}_{o}-\hat{\bar{x}}_{o}$. If there exists symmetric positive definite matrices $Q=Q^{\top} \succ 0$ and $K=K^{\top} \succ 0$, such that the following linear matrix inequalities (LMI)

$$
\left[\begin{array}{cc}
\bar{A}_{o}^{\top} Q+Q \bar{A}_{o} & -Q \\
-Q & 0
\end{array}\right] \prec 0, \quad\left[\begin{array}{cc}
\gamma^{2} L^{\top} L I & 0 \\
0 & -\mathbb{I}-K
\end{array}\right] \succeq 0,
$$

yield a feasible solution, then the estimation error $\tilde{\bar{x}}_{o}$ converges to zero asymptotically.

Proof: The dynamics for error system $\tilde{\bar{x}}_{o}$ is given by

$$
\dot{\bar{x}}_{o}=\bar{A}_{o} \tilde{\bar{x}}_{o}-L \tilde{y} .
$$

where $\tilde{y}=y-\hat{y}$. We choose the Lyapunov function candidate

$$
W(t)=\tilde{\bar{x}}_{o}^{\top} Q \tilde{\bar{x}}_{o}, \quad Q=Q^{\top} \succ 0,
$$

and the time derivative of $W$ along the trajectory of $\tilde{\bar{x}}_{o}$ is

$$
\begin{aligned}
\dot{W} & =\dot{\bar{x}}_{o}^{\top} Q \tilde{\bar{x}}_{o}+\tilde{\bar{x}}_{o}^{\top} Q \dot{\overline{\bar{x}}}_{o} \\
& =\tilde{\bar{x}}_{o}^{\top}\left(\bar{A}^{\top} Q+Q \bar{A}\right) \tilde{\bar{x}}_{o}-(L \tilde{y})^{\top} Q \tilde{\bar{x}}_{o}-\tilde{\bar{x}}_{o}^{\top} Q(L \tilde{y}) \\
& =\left[\begin{array}{cc}
\tilde{\bar{x}}_{o}^{\top} & z^{\top}
\end{array}\right]\left[\begin{array}{cc}
\bar{A}^{\top} Q+Q \bar{A} & -Q \\
-Q & 0
\end{array}\right]\left[\begin{array}{c}
\tilde{\bar{x}}_{o} \\
z
\end{array}\right],
\end{aligned}
$$

where $z=L \tilde{y}$. For $\dot{W}$ to be negative definite, we require the first condition in (29). Moreover, according to Remark 1, Lipschitz continuity with respect to $\bar{x}_{o}$ produces $\|\tilde{y}\| \leq \gamma\left\|\tilde{\bar{x}}_{o}\right\|$. Applying Cauchy-Schwarz inequality [14] to $z=L \tilde{y}$ yields

$$
\|z\| \leq\|L\|\|\tilde{y}\| \leq \gamma\|L\|\left\|\tilde{\bar{x}}_{o}\right\|,
$$

which can be alternatively written as $z^{\top} z \leq \gamma^{2} L^{\top} L \tilde{\bar{x}}_{o}^{\top} \tilde{\bar{x}}_{o}$. We can add a tuning term to form a linear matrix inequality,

$$
z^{\top} z-\tilde{\bar{x}}_{o}^{\top}\left(\gamma^{2} L^{\top} L I\right) \tilde{\bar{x}}_{o} \leq-z^{\top} K z,
$$

where $K \succ 0$ is a positive definite matrix chosen by the designer. Reformulating (34) into

$$
\left[\begin{array}{cc}
\tilde{\bar{x}}_{o}^{\top} & z^{\top}
\end{array}\right]\left[\begin{array}{cc}
\gamma^{2} L^{\top} L I & 0 \\
0 & -\mathbb{I}-K
\end{array}\right]\left[\begin{array}{c}
\tilde{\bar{x}}_{o} \\
z
\end{array}\right] \succeq 0 .
$$

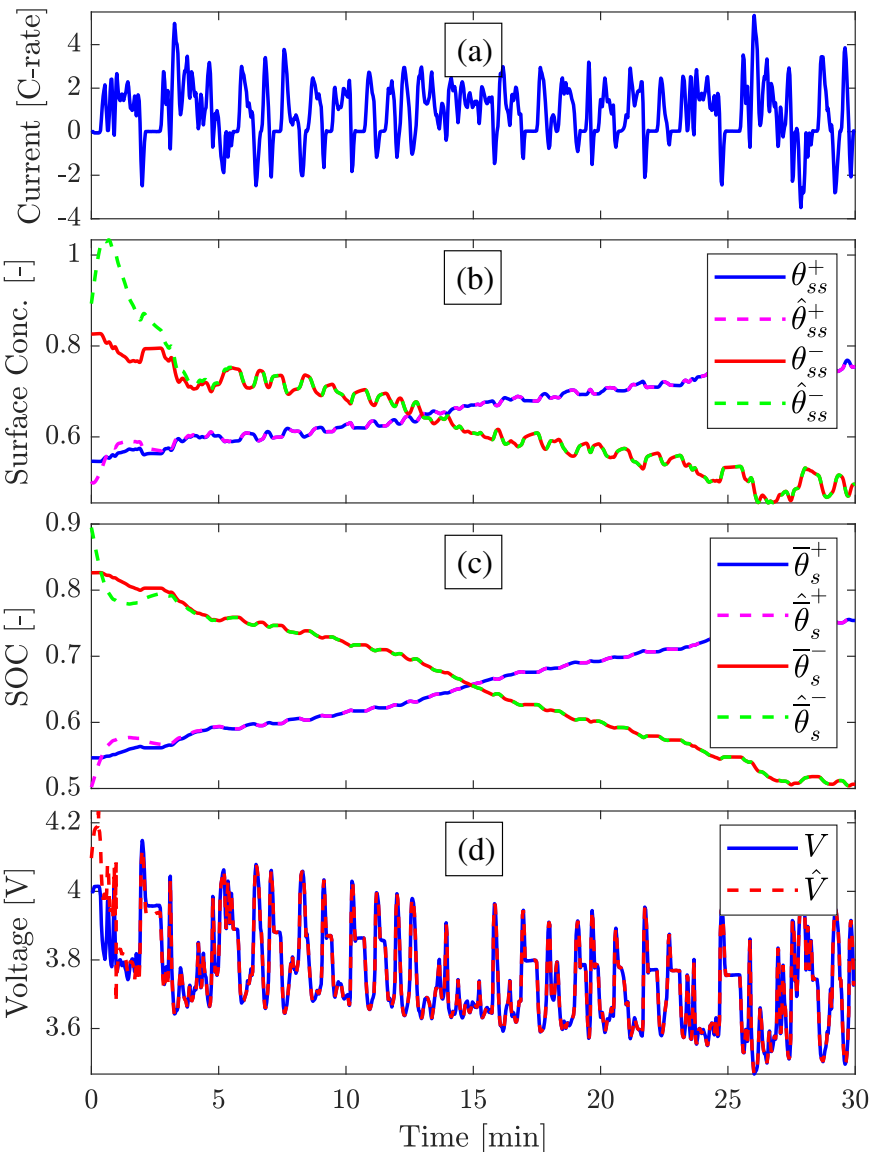

Fig. 1. Observer results for SPM via a nonlinear observer design in a transformed coordinate using observability decomposition, using an UDDS drive cycle in simulation. (a) applied current; (b). surface concentration; (c) volume-averaged concentration (SOC); (d). voltage.

Therefore, for $W$ to converge to zero asymptotically, the matrix inequalities in (29) need to be satisfied.

\section{SiMULATIONS}

In this section, we present simulation results to demonstrate the performance of the proposed observer for electrode-level state estimation. The input current in Fig. 1(a) is generated from an Urban Dynamometer Driving Schedule (UDDS) drive cycle, to imitate a practical electric vehicle driving pattern. The considered battery chemistry is graphite/lithium cobalt oxide, whose model parameters can be found in [15]. For all simulations presented in this section, the state estimates are initialized at incorrect values. The observer (28) is implemented numerically on the observable $\bar{x}_{o^{-}}$-system (23). The observer gain is selected based upon the LMI condition (29) in Theorem 2. The observer gain that satisfies condition (29) is $L=\left[\begin{array}{lllll}1.90 & 1.26 & 0.32 & 0.014 & -0.0020\end{array}\right]^{\top}$. The normalized surface concentration estimates are initialized at 0.50 and 0.89 for positive and negative electrode, respectively, and each with $8.1 \%$ error against the plant model, to validate the convergence property. Figure 1(b) presents the temporal evolution of the surface concentration estimates, with an asymptotic convergence time less than 180 seconds. After the initial transient period, the root mean squared errors (RMSE) 
between the (normalized) estimates and the plant models are $2 \times 10^{-4}$ and $1.2 \times 10^{-3}$, respectively for positive and negative electrodes. Similarly, the normalized volume-averaged concentrations (namely the electrode-level SOCs) are plotted in Fig. 1(c), with RMSE being $1.5 \times 10^{-4}$ and $2.4 \times 10^{-3}$ after the initial transient. With the appropriately selected gain, as detailed in Theorem 2, the voltage estimates also converge to their true values from the plant model asymptotically, shown in Fig. 1(d), with RMSE value at $0.15 \mathrm{mV}$.

\section{CONCLUSION}

This paper presents a local observability analysis for a single particle model (SPM) with dynamics of both electrodes. The SPM is first reduced in frequency domain with a Padé approximation. The state-space realization, which has been proven to be locally unobservable, is decomposed according to the Kalman decomposition. This coordinate transformation permits a separation of observable components from the unobservable sub-system. By assuming knowledge of the total moles of solid-phase lithium, this conservation law can be integrated into the transformation matrix, enabling an inverse transformation to reconstruct the essential electrochemical states of both electrodes. Subsequently, an asymptotically convergent state observer is designed for the observable subsystem in the transformed coordinate. The proposed electrodelevel observer retains a simple structure compared to other works with complex interconnected observers. This work addresses the local observability issues for SPM, and paves a path for state estimation for both electrodes. Future works will examine the practical performances of the proposed framework on real battery experimental data.

\section{REFERENCES}

[1] N. A. Chaturvedi, R. Klein, J. Christensen, J. Ahmed, and A. Kojic, "Algorithms for advanced battery-management systems," IEEE Control systems magazine, vol. 30, no. 3, pp. 49-68, 2010.

[2] M. A. Hannan, M. S. H. Lipu, A. Hussain, and A. Mohamed, "A review of lithium-ion battery state of charge estimation and management system in electric vehicle applications: Challenges and recommendations," Renewable and Sustainable Energy Reviews, vol. 78, pp. 834-854, 2017.

[3] K. A. Severson, P. M. Attia, N. Jin, N. Perkins, B. Jiang, Z. Yang, M. H. Chen, M. Aykol, P. K. Herring, D. Fraggedakis, et al., "Data-driven prediction of battery cycle life before capacity degradation," Nature Energy, vol. 4, no. 5, pp. 383-391, 2019.

[4] S. Li, H. He, C. Su, and P. Zhao, "Data driven battery modeling and management method with aging phenomenon considered," Applied Energy, vol. 275, p. 115340, 2020.

[5] X. Hu, S. Li, and H. Peng, "A comparative study of equivalent circuit models for li-ion batteries," Journal of Power Sources, vol. 198, pp. 359367, 2012.

[6] D. Zhang, S. Dey, H. E. Perez, and S. J. Moura, "Remaining useful life estimation of lithium-ion batteries based on thermal dynamics," in 2017 American Control Conference (ACC), pp. 4042-4047, IEEE, 2017.

[7] M. Doyle, T. F. Fuller, and J. Newman, "Modeling of galvanostatic charge and discharge of the lithium/polymer/insertion cell," Journal of the Electrochemical Society, vol. 140, no. 6, pp. 1526-1533, 1993.

[8] D. Zhang, S. Dey, and S. J. Moura, "Lithium-ion battery state estimation for a single particle model with intercalation-induced stress," in 2018 Annual American Control Conference (ACC), pp. 2294-2299, IEEE, 2018.

[9] S. J. Moura, F. B. Argomedo, R. Klein, A. Mirtabatabaei, and M. Krstic, "Battery state estimation for a single particle model with electrolyte dynamics," IEEE Transactions on Control Systems Technology, vol. 25, no. 2, pp. 453-468, 2016.
[10] D. Di Domenico, A. Stefanopoulou, and G. Fiengo, "Lithium-ion battery state of charge and critical surface charge estimation using an electrochemical model-based extended kalman filter," Journal of Dynamic Systems, Measurement, and Control, vol. 132, no. 6, pp. 061302.1$061302.11,2010$.

[11] A. Bartlett, J. Marcicki, S. Onori, G. Rizzoni, X. G. Yang, and T. Miller, "Electrochemical model-based state of charge and capacity estimation for a composite electrode lithium-ion battery," IEEE Transactions on Control Systems Technology, vol. 24, no. 2, pp. 384-399, 2016.

[12] X. Hu, D. Cao, and B. Egardt, "Condition monitoring in advanced battery management systems: Moving horizon estimation using a reduced electrochemical model," IEEE/ASME Transactions on Mechatronics, vol. 23, no. 1, pp. 167-178, 2017.

[13] D. Zhang, S. Dey, L. D. Couto, and S. J. Moura, "Battery adaptive observer for a single-particle model with intercalation-induced stress," IEEE transactions on control systems technology, 2019.

[14] S. Dey, B. Ayalew, and P. Pisu, "Nonlinear robust observers for state-ofcharge estimation of lithium-ion cells based on a reduced electrochemical model," IEEE Transactions on Control Systems Technology, vol. 23, no. 5, pp. 1935-1942, 2015.

[15] S. J. Moura, N. A. Chaturvedi, and M. Krstić, "Adaptive partial differential equation observer for battery state-of-charge/state-of-health estimation via an electrochemical model," Journal of Dynamic Systems, Measurement, and Control, vol. 136, no. 1, pp. 011015-1-011015-11, 2014.

[16] S. Dey and B. Ayalew, "Real-time estimation of lithium-ion concentration in both electrodes of a lithium-ion battery cell utilizing electrochemical-thermal coupling," Journal of Dynamic Systems, Measurement, and Control, vol. 139, no. 3, pp. 031007-031007-10, 2017.

[17] S. Pannala, P. Valecha, P. Mohtat, J. B. Siegel, and A. G. Stefanopoulou, "Improved battery state estimation under parameter uncertainty cause by aging using expansion measurements," in 2021 American Control Conference (ACC), IEEE, 2021.

[18] S. Dey, Y. Shi, K. Smith, A. Colclasure, and X. Li, "From battery cell to electrodes: Real-time estimation of charge and health of individual battery electrodes," IEEE Transactions on Industrial Electronics, pp. 1$1,2019$.

[19] S. Sattarzadeh, S. Dey, A. Colclasure, and K. Smith, "Addressing the observability problem in batteries: Algorithm design for electrode-level charge and health estimation," in 2020 American Control Conference (ACC), pp. 1131-1136, IEEE, 2020.

[20] L. Camacho-Solorio, M. Krstic, R. Klein, A. Mirtabatabaei, and S. J. Moura, "State estimation for an electrochemical model of multiplematerial lithium-ion batteries," in Dynamic Systems and Control Conference, vol. 50695, p. V001T08A004, American Society of Mechanical Engineers, 2016.

[21] R. Klein, N. A. Chaturvedi, J. Christensen, J. Ahmed, R. Findeisen, and A. Kojic, "Electrochemical model based observer design for a lithiumion battery," IEEE Transactions on Control Systems Technology, vol. 21, no. 2, pp. 289-301, 2013.

[22] J. C. Forman, S. Bashash, J. L. Stein, and H. K. Fathy, "Reduction of an electrochemistry-based Li-ion battery model via quasi-linearization and padé approximation," Journal of the Electrochemical Society, vol. 158, no. 2, p. A93, 2010.

[23] J. Marcicki, M. Canova, A. T. Conlisk, and G. Rizzoni, "Design and parametrization analysis of a reduced-order electrochemical model of graphite/ $\mathrm{LiFePO}_{4}$ cells for $\mathrm{SOC} / \mathrm{SOH}$ estimation," Journal of Power Sources, vol. 237, pp. 310-324, 2013.

[24] H. Kimura, Chain-scattering approach to $H \infty$-control. Springer Science \& Business Media, 2012.

[25] L. Weiss and R. Kalman, "Contributions to linear system theory," International Journal of Engineering Science, vol. 3, no. 2, pp. 141171, 1965.

[26] R. E. Kalman, "Mathematical description of linear dynamical systems," Journal of the Society for Industrial and Applied Mathematics, Series A: Control, vol. 1, no. 2, pp. 152-192, 1963.

[27] H. J. Marquez, Nonlinear control systems. John Wiley \& Sons., 2003.

[28] R. Rajamani, "Observers for lipschitz nonlinear systems," IEEE transactions on Automatic Control, vol. 43, no. 3, pp. 397-401, 1998. 